\title{
Características clínicas e laboratoriais associadas à nefroangioesclerose hipertensiva confirmada por biópsia renal
}

\author{
Clinical and laboratorial characteristics associated with biopsy \\ proved hypertensive nephroangiosclerosis
}

\begin{abstract}
Autores
Carolina Hernandez D'Oliveira $^{1}$

Luis Cuadrado Martin ${ }^{1}$

Rosa Marlene Viero ${ }^{1}$

Camila Farias Cerolli ${ }^{1}$

Cinthia Esbrille de

Moraes $^{1}$

Vanessa dos Santos

Silva ${ }^{1}$

Francisco Habermann ${ }^{1}$

Roberto Jorge da Silva Franco $^{1}$

${ }^{1}$ Faculdade de Medicina de Botucatu da Universidade Estadual Paulista "Júlio de Mesquita Filho"- UNESP.
\end{abstract}

Data de submissão: 17/02/2011 Data de aprovação: 05/06/2011

Correspondência para: Luis Cuadrado Martin Departamento de Clínica Médica da Faculdade de Medicina de Botucatu da UNESP

Distrito de Rubião Júnior, s/n Botucatu (SP) - Brasil

CEP: 18618-970

E-mail: cuadrado@fmb. unesp.br

Suporte financeiro: $\mathrm{PIBIC/CNPQ}$.

O referido estudo foi realizado no Centro de Hipertensão Arterial da Faculdade de Medicina de Botucatu da UNESP.

Os autores declaram a inexistência de conflitos de interesse.

\section{Resumo}

Introdução: A nefroangioesclerose hipertensiva é importante causa de doença renal crônica com necessidade de diálise. As características que distinguem um portador de hipertensão arterial que evolui com nefroangioesclerose de outro que mantém função renal estável não são bem estabelecidas, devido à dificuldade em assegurar que os portadores daquela doença não sejam, na verdade, portadores de glomerulopatias ou outras doenças renais confundíveis. Dessa maneira, o objetivo deste trabalho foi identificar características clínicas ou laboratoriais que distingam os pacientes que desenvolveram doença renal crônica a partir da hipertensão, confirmada por biópsia renal, daqueles que, mesmo apresentando hipertensão arterial, não desenvolveram nefroangioesclerose. Métodos: Realizou-se comparação retrospectiva de dados clínicos e laboratoriais de 15 portadores de nefroangioesclerose hipertensiva confirmada por biópsia renal e 15 hipertensos oriundos do ambulatório do Centro de Hipertensão Arterial, cuja ausência de nefroangioesclerose foi definida pela ausência de proteinúria. Os grupos foram pareados quanto à idade e gênero. Resultados: Dentre as variáveis avaliadas, tempo de hipertensão arterial, pressão de pulso, glicemia, ácido úrico, creatinina e frequência de uso de diuréticos e simpatolíticos diferiram estatisticamente entre os dois grupos. Todas essas variáveis apresentaram valores maiores no grupo com nefroangioesclerose hipertensiva. Conclusão: O presente estudo associa a nefroangioesclerose hipertensiva, confirmada por biópsia, com alterações metabólicas, duração e intensidade da hipertensão e corrobora a ideia de que a prevenção primária da hipertensão arterial, postergando o seu início, o controle pressórico mais estrito,

\section{Abstract}

Introduction: Hypertensive nephroangiosclerosis is a major cause of chronic kidney disease requiring dialysis. Clinical characteristics that distinguish a patient with hypertension that evolves to nephroangiosclerosis from another that keeps stable renal function are not well established because of the difficulty in ensuring that the carriers of that disease are not actually suffering from glomerulonephritis or other kidney diseases. Thus, our objective was to identify clinical or laboratory features that distinguish the patients who developed chronic renal failure from hypertension, confirmed by renal biopsy, of those who, even with arterial hypertension, did not develop nephroangiosclerosis. Methods: We conducted a retrospective comparison of clinical and laboratory data of 15 patients with hypertensive nephroangiosclerosis confirmed by renal biopsy and 15 hypertensive patients from the outpatient clinic of the Hypertension Center, whose lack of nephroangiosclerosis was defined as absence of proteinuria. The groups were matched for age and gender. Results: Among the evaluated variables, duration of hypertension, pulse pressure, blood glucose, uric acid, creatinine and frequency of use of diuretics and sympatholytic differed statistically between the two groups. All these variables were higher in nephroangiosclerosis patients. Conclusion: This study links biopsy proven hypertensive nephroangiosclerosis with metabolic features, hypertension intensity and duration, corroborating the idea that primary prevention of hypertension, postponing its initiation, a more intensive hemodynamic control (when hypertension is 
quando a hipertensão já está estabelecida, bem como o controle metabólico têm a potencialidade de prevenir o desenvolvimento de nefroangioesclerose hipertensiva.

Palavras-chave: Hipertensão. Falência renal crônica. Hiperuricemia. Creatinina. Hiperglicemia. well established) and metabolic control of these patients have the potential to prevent hypertensive nephroangiosclerosis.

Keywords: Hypertension. Kidney failure. Chronic. Hyperuricemia. Creatinine. Hyperglycemia.

\section{INTRODUÇÃO}

A prevalência de insuficiência renal crônica com necessidade de diálise tem aumentado dramaticamente em todo o mundo, bem como em nosso meio. ${ }^{1}$ A hipertensão arterial (HA) é doença altamente prevalente, ocorrendo em torno de $30 \%$ da população brasileira. ${ }^{1}$ É a HA o fator de risco de morte preponderante no mundo atual. As principais causas de óbito entre hipertensos são as doenças cardiovasculares, principalmente o acidente vascular encefálico e o infarto do miocárdio. ${ }^{2}$

Dentre todos os casos de HA, poucos desenvolverão nefroangioesclerose hipertensiva com evolução para insuficiência renal. ${ }^{3}$ Entretanto, como o número de hipertensos na população é muito elevado, ainda que uma pequena fração evolua para insuficiência renal, haverá um número expressivo de pacientes que atingirão o estádio de diálise por nefroangioesclerose hipertensiva quando analisada em números absolutos. Assim, a nefroangioesclerose hipertensiva é a segunda causa mais frequente de insuficiência renal tanto nos países desenvolvidos como nos países em desenvolvimento. ${ }^{3}$ Além disso, com o aumento da expectativa de vida, o número de hipertensos aumentou significativamente, com maior preocupação com o desenvolvimento de insuficiência renal por nefroangioesclerose hipertensiva.

Historicamente, a HA foi classificada, quanto ao seu caráter, em benigna e maligna. A hipertensão maligna manifesta-se por elevação intensa da pressão arterial (PA) com deterioração progressiva dos órgãos-alvo, mormente os rins, caracterizada na anatomia patológica por endarterite obliterante e arteriolite necrotizante. ${ }^{4} \mathrm{~A}$ hipertensão dita "benigna" caracteriza-se pela hialinose arteriolar e, até pouco tempo atrás, considerava-se uma entidade clínica sem potencial de evolução para insuficiência renal. Hoje, sabe-se que a nefroangioesclerose hipertensiva "benigna" é causa frequente de insuficiência renal pelos motivos já expostos acima. ${ }^{5}$

Tendo em vista que, dentre muitos hipertensos, apenas uma pequena fração evolui para insuficiência renal crônica, não há como prever qual paciente poderá apresentar essa ominosa doença. Conhecer características clínicas associadas ao desenvolvimento de insuficiência renal reveste-se de importância, uma vez que essa informação pode dirigir possíveis programas e tratamentos preventivos baseados no padrão estabelecido.

Estudos que avaliaram esses fatores de evolução para insuficiência renal em hipertensos não determinaram de maneira inequívoca a etiologia da doença renal. Assim, os pacientes que atingiram o desfecho nesses estudos poderiam apresentar várias outras patologias renais que não a própria nefroangioesclerose hipertensiva. ${ }^{6}$

Dessa maneira, o objetivo deste trabalho foi identificar características clínicas ou laboratoriais que distingam os pacientes que desenvolveram insuficiência renal crônica a partir da hipertensão (confirmada por biópsia renal) daqueles que, mesmo apresentando hipertensão arterial, não desenvolveram nefroangioesclerose hipertensiva.

\section{Métodos}

Foram analisados, retrospectivamente, os dados clínicos e laboratoriais de 15 pacientes consecutivos que realizaram biópsias renais no Laboratório de Anatomia Patológica do Departamento de Patologia da Faculdade de Medicina de Botucatu da Universidade Estadual Paulista "Júlio de Mesquita Filho" (UNESP), provenientes de pacientes cujo diagnóstico anatomopatológico firmado foi nefroangioesclerose hipertensiva. O critério utilizado para realização de biópsia renal nesses pacientes foi a presença de proteinúria superior a $2,0 \mathrm{~g}$ em 24 horas ou deterioração progressiva da função renal. Realizouse comparação entre pacientes com diagnóstico anatomopatológico de nefroangioesclerose hipertensiva e pacientes hipertensos oriundos do ambulatório do Centro de Hipertensão Arterial (CHA) pareados quanto à idade e gênero (Grupo Controle; $n=15$ ). Os pacientes foram avaliados no período de agosto de 1970 a junho de 2006. O tempo transcorrido entre a avaliação clínica e a inclusão na pesquisa foi de 
$11 \pm 2,3$ anos no grupo com nefroesclerose hipertensiva e $12 \pm 8,6$ anos no Grupo Controle.

A presença de nefroangioesclerose hipertensiva foi definida histologicamente como esclerose glomerular e predomínio de lesões vasculares, quais sejam: hialinose arteriolar, hipertrofia da camada média e fibrose intimal. Foram excluídos os pacientes que apresentavam qualquer sinal de doença glomerular específica, primária ou secundária a outras doenças que não a HA, nefrite tubulointersticial, nefropatia diabética e aqueles com lesões compatíveis com hipertensão acelerada/maligna. A rotina de avaliação histológica do nosso serviço consta de microscopia óptica e imunofluorescência.

Os pacientes do grupo controle preencheram os seguintes critérios de inclusão: tratamento no ambulatório do Centro de Hipertensão Arterial (CHA) com pressão arterial sistólica (PAS) $\geq 140 \mathrm{mmHg}$ e pressão arterial diastólica (PAD) $\geq 90 \mathrm{mmHg}$ ou persistente necessidade de anti-hipertensivos, ausência de doença renal definida como filtração glomerular superior a $60 \mathrm{~mL} / \mathrm{min}$ e ausência de proteinúria. ${ }^{3}$ Esses pacientes não apresentavam nenhum dos seguintes critérios de exclusão: portadores de estenoses da artéria renal, outras causas de hipertensão secundária, doença imunomediada, falência cardíaca, doença hepática, obstrução urinária, gravidez e doença renal policística.

Os prontuários dos pacientes, tanto do grupo controle como do grupo com nefroangioesclerose hipertensiva, foram analisados e deles foram obtidas as seguintes variáveis clínicas: idade, gênero, etnia, tempo de evolução da HA, tabagismo, presença de diabetes, peso, altura, pressão arterial, medicações utilizadas e dose, número de classes de anti-hipertensivos, creatinina, filtração glomerular estimada pela fórmula de Cockcroft-Gault, colesterol, triglicérides, HDL-colesterol, ácido úrico, glicemia, potássio e dados do exame de urina tipo I. Proteinúria de 24 horas foi avaliada no grupo com nefroangioesclerose. Avaliou-se também a presença de hipertensão resistente, que foi definida como a presença de pressão arterial igual ou superior a 140 × $90 \mathrm{mmHg}$ em vigência de três classes distintas de drogas anti-hipertensivas em dose máxima, sendo uma delas diurético. O valor representativo da pressão arterial de cada paciente foi a média de todas as aferições disponíveis no prontuário do paciente que foram realizadas nas consultas médicas da disciplina de Nefrologia, nas quais se buscava avaliar a pressão arterial de acordo com as recomendações das Diretrizes Brasileiras de Hipertensão. ${ }^{1}$

Os dados das variáveis contínuas e paramétricas foram expressos em média \pm desvio padrão; as variáveis não paramétricas foram expressas em mediana (intervalo interquartílico) e as variáveis categóricas foram expressas em frequências. Os grupos foram comparados quanto aos dados paramétricos pelo teste $t$ de Student para amostras independentes e pelo teste de Mann-Whitney quanto aos dados não paramétricos. As variáveis categóricas foram comparadas pelo teste do $\chi^{2}$. Foram considerados estatisticamente significantes os valores de $\mathrm{p}<0,05$.

\section{Resultados}

As variáveis idade, altura, peso, índice de massa corpórea, PAS, PAD, colesterol total, HDLcolesterol, triglicérides e potássio não apresentaram diferença estatística, comparando-se os grupos com nefroangioesclerose hipertensiva e controle hipertensos sem proteinúria (Tabela 1). Como os dois grupos foram pareados por idade, as médias e os desvios padrão das idades foram semelhantes, sendo $55,86 \pm 10,11$ e 55,27 $\pm 9,98$ anos, respectivamente. Quanto à altura, a média para o grupo com nefroangioesclerose foi de 1,67 $\pm 0,12$ e, para o Grupo Controle, 1,68 $\pm 0,11$. A média do peso para o grupo com nefroangioesclerose foi de $74,25 \pm 15,75 \mathrm{~kg}$ e, no Grupo Controle, foi de 75,56 $\pm 16,35 \mathrm{~kg}$. A média do índice de massa corpórea foi $27,45 \pm$ $4,33 \mathrm{~kg} / \mathrm{m}^{2}$ para o grupo com nefroangioesclerose e $26,68 \pm 4,43 \mathrm{~kg} / \mathrm{m}^{2}$ para o grupo controle. A PAS para o grupo com nefroangioesclerose foi $170,21 \pm 32,28 \mathrm{~mm} \mathrm{Hg}$ e para o grupo controle foi $153,73 \pm 26,10 \mathrm{~mm} \mathrm{Hg}$. O grupo com nefroangioesclerose apresentou PAD de 103,37 $\pm 19,78 \mathrm{mmHg}$ e o Grupo Controle 103,20 $\pm 19,64 \mathrm{mmHg}$. Para colesterol total, o grupo com nefroangioesclerose apresentou uma mediana de $205 \mathrm{mg} / \mathrm{dL}$ (178 - 255) e o Grupo Controle, 226 mg/dL (202 - 243). Para HDL-colesterol, no grupo com nefroangioesclerose, obteve-se 33 mg/dL (23 - 45) e, no Grupo Controle, $38 \mathrm{mg} / \mathrm{dL}$ (31-45). Para os triglicérides, a mediana do grupo com nefroangioesclerose foi de 222 mg/dL (146 - 430) e a do Grupo Controle foi 180 $\mathrm{mg} / \mathrm{dL}$ (134 - 276). A média para o potássio foi de $4,49 \pm 0,62 \mathrm{mg} / \mathrm{dL}$ para o grupo com nefroangioesclerose e de 4,28 $\pm 0,52 \mathrm{mg} / \mathrm{dL}$ para o Grupo Controle. A proteinúria foi de 2,55 $\pm 0,69 \mathrm{~g}$ em 24 horas no grupo com nefroangioesclerose e não foi 


\begin{tabular}{|c|c|c|c|c|}
\hline \multirow[t]{2}{*}{ Tabela 1} & \multicolumn{4}{|c|}{$\begin{array}{l}\text { VARIÁVEIS CONTÍNUAS COMPARANDO PACIENTES COM NEFROANGIOESCLEROSE HIPERTENSIVA E GRUPO } \\
\text { CONTROLE DE HIPERTENSOS PAREADO POR IDADE E SEXO }\end{array}$} \\
\hline & & $\begin{array}{l}\text { Grupo Nefroangioesclerose } \\
\qquad(\mathrm{n}=15)\end{array}$ & $\begin{array}{l}\text { Grupo Controle } \\
\qquad(n=15)\end{array}$ & $\mathrm{p}$ \\
\hline \multicolumn{2}{|l|}{ Idade (anos) } & $55,86 \pm 10,11$ & $55,27 \pm 9,98$ & 0,87 \\
\hline \multicolumn{2}{|c|}{ Tempo de HA (anos) } & $10(3,3-16,0)$ & $2(0,0-6,3)$ & 0,02 \\
\hline \multicolumn{2}{|c|}{ Altura (m) } & $1,67 \pm 0,125$ & $1,68 \pm 0,114$ & 0,83 \\
\hline \multicolumn{2}{|l|}{ Peso $(\mathrm{kg})$} & $74,25 \pm 15,75$ & $75,56 \pm 16,35$ & 0,83 \\
\hline \multicolumn{2}{|l|}{ IMC (kg/m²) } & $27,45 \pm 4,33$ & $26,68 \pm 4,43$ & 0,67 \\
\hline \multicolumn{2}{|c|}{ PAS (mmHg) } & $170,21 \pm 32,28$ & $153,73 \pm 26,10$ & 0,14 \\
\hline \multicolumn{2}{|c|}{ PAD $(\mathrm{mmHg})$} & $103,37 \pm 19,78$ & $103,20 \pm 19,64$ & 0,98 \\
\hline \multicolumn{2}{|l|}{$\mathrm{PP}(\mathrm{mmHg})$} & $66,84 \pm 19,94$ & $50,53 \pm 17,78$ & 0,03 \\
\hline \multicolumn{2}{|c|}{ Número de classes de anti-hipertensivos } & $2(2,00-2,75)$ & $1(0,25-2,00)$ & 0,01 \\
\hline \multicolumn{2}{|c|}{ Creatinina $(\mathrm{mg} / \mathrm{dL})$} & $4,47 \pm 3,70$ & $1,18 \pm 0,43$ & $<0,01$ \\
\hline \multicolumn{2}{|c|}{ Filtração glomerular (mL/min) } & $27,5 \pm 17,9$ & $85,0 \pm 31,9$ & $<0,01$ \\
\hline \multicolumn{2}{|c|}{ Ácido úrico (mg/dL) } & $8,36 \pm 2,18$ & $6,00 \pm 2,14$ & 0,02 \\
\hline \multicolumn{2}{|c|}{ Colesterol total (mg/dL) } & 205 (178-255) & $226(202-243)$ & 0,58 \\
\hline \multicolumn{2}{|c|}{ HDL-colesterol (mg/dL) } & $33(23-45)$ & $38(31-45)$ & 0,56 \\
\hline \multicolumn{2}{|c|}{ Triglicérides (mg/dL) } & $222(146-430)$ & $180(134-276)$ & 0,26 \\
\hline \multicolumn{2}{|c|}{ Potássio (mg/dL) } & $4,49 \pm 0,62$ & $4,28 \pm 0,52$ & 0,43 \\
\hline \multicolumn{2}{|c|}{ Glicemia (mg/dL) } & $119,95 \pm 32,82$ & $99,69 \pm 6,00$ & 0,04 \\
\hline
\end{tabular}

HA: hipertensão arterial; IMC: índice de massa corpórea; PAS: pressão arterial sistólica; PAD: pressão arterial diastólica; PP: pressão de pulso.

quantificada no Grupo Controle por este não apresentar sequer traços de proteinúria à urina $\mathrm{I}$.

As seguintes variáveis apresentaram diferença estatística entre os dois grupos: tempo de HA, número de classes de anti-hipertensivos empregada, pressão de pulso, creatinina, filtração glomerular, ácido úrico e glicemia. Assim, para essas variáveis, o grupo com nefroangioesclerose apresentou valores maiores que o Grupo Controle (Tabela 1). Para tempo de HA, o grupo com nefroangioesclerose apresentou $(3,3$ - 16,0) 10 anos e o Grupo Controle, $(0,0$ - 6,3) 2 anos. O número de classes de anti-hipertensivos empregado foi de $2(2,00-2,75)$ no grupo com nefroangioesclerose e $1(0,25$ - 2,00) no Grupo Controle. Para a pressão de pulso $(\mathrm{PP})$, o grupo com nefroangioesclerose apresentou 66,84 $\pm 19,94 \mathrm{mmHg}$ e o Grupo Controle, $50,53 \pm 17,78 \mathrm{mmHg}$. A creatinina do grupo com nefroangioesclerose foi de 4,47 $\pm 3,70 \mathrm{mg} / \mathrm{dL}$ e do Grupo Controle foi de 1,18 $\pm 0,43 \mathrm{mg} / \mathrm{dL}$. A filtração glomerular do Grupo Controle foi de $85,0 \pm 31,9 \mathrm{~mL} / \mathrm{min}$ e do grupo com nefroangioesclerose foi de $27,5 \pm 17,9 \mathrm{~mL} / \mathrm{min}$. Para o ácido úrico, a média do grupo com nefroangioesclerose foi 8,36 $\pm 2,18 \mathrm{mg} / \mathrm{dL}$ e a do Grupo Controle foi $6,00 \pm 2,14 \mathrm{mg} / \mathrm{dL}$. Para glicemia, a média do grupo com nefroangioesclerose foi $119,95 \pm 32,82$ e do Grupo Controle foi 99,69 $\pm 6,00$ (Tabela 1 ).
Quanto às variáveis categóricas, comparando os grupos com nefroangioesclerose hipertensiva e controle de hipertensos sem proteinúria, não se obteve significância estatística em nenhuma das variáveis avaliadas, exceto quanto ao uso de diuréticos e simpatolíticos. A proporção entre o sexo feminino e masculino foi de 2:13 tanto para o grupo com nefroangioesclerose como para o Grupo Controle, já que eles foram pareados por sexo e idade. Já a proporção entre brancos e não brancos foi de 13:2 no grupo com nefroangioesclerose e 14:1 no Grupo Controle. A proporção entre tabagistas, não tabagistas e ex-tabagistas para o grupo com nefroangioesclerose foi de 2:6:6 e para o Grupo Controle foi de 3:5:4, respectivamente. A proporção de diabéticos e não diabéticos foi de 3:10 para o grupo com nefroangioesclerose e 2:11 para o Grupo Controle, respectivamente. Para o uso ou não de bloqueador de cálcio, o grupo com nefroangioesclerose obteve uma proporção de 5:10 e o Grupo Controle, uma proporção de 1:14, respectivamente. Para o uso ou não de beta-bloqueador, a proporção para o grupo com nefroangioesclerose foi de 3:12 e para o Grupo Controle foi de 2:13, respectivamente. Para o uso ou não de vasodilatador, a proporção para o grupo com nefroangioesclerose foi de 1:14 e para o Grupo Controle foi de 0:15, respectivamente. Para o uso ou não de bloqueadores do 
receptor de angiotensina (BRA), o grupo com nefroangioesclerose mostrou uma proporção de 2:13 e o Grupo Controle, uma proporção de 1:14, respectivamente. Para o uso ou não de inibidores da enzima conversora do angiotensinogênio (IECA), o grupo com nefroangioesclerose mostrou uma proporção de 4:11 e o Grupo Controle, uma proporção de 5:10, respectivamente. Para o uso ou não do duplo bloqueio com BRA e IECA, os dois grupos obtiveram a mesma proporção de 1:14. O uso ou não de diuréticos e simpatolíticos foram as únicas variáveis que obtiveram significância estatística. Em relação ao uso ou não de diuréticos, a proporção para o grupo com nefroangioesclerose foi de 13:2 e para o Grupo Controle foi de 8:7. Em relação ao uso ou não de simpatolíticos, a proporção para o grupo com nefroangioesclerose foi de 7:8 e para o Grupo Controle, 2:13 (Tabela 2).

No grupo com nefroangioesclerose hipertensiva, quatro pacientes apresentaram anormalidades do sedimento urinário: dois apresentaram apenas hematúria microscópica, um apresentou apenas leucocitúria e um apresentou hematúria microscópica e leucocitúria concomitantes. Nenhum paciente do Grupo Controle apresentou anormalidades do sedimento urinário.

$\mathrm{O}$ uso de simpatolíticos foi mais frequente no grupo com nefroangioesclerose em relação ao Grupo Controle: $7: 8$ versus $2: 13$, respectivamente. $\mathrm{O}$ uso de diuréticos foi mais frequente no grupo com nefroangioesclerose em relação ao Grupo Controle: 13:2 versus 8:7, respectivamente. Diuréticos de alça foram mais empregados no grupo com nefroesclerose.

No grupo com nefroangioesclerose, 14 dos 15 pacientes apresentavam-se com pressão arterial superior a 140 × $90 \mathrm{mmHg}$. Destes 14, 6 apresentavam todos os critérios expostos para hipertensão refratária, ou seja, 3 classes em dose máxima, sendo uma delas diurético; dos oito restantes, um não utilizava diurético, três utilizavam apenas duas classes de drogas e quatro, apesar de utilizarem três classes de anti-hipertensivos, não utilizavam as doses máximas preconizadas. No Grupo Controle, 10 dos 15 pacientes apresentavam-se com pressão arterial superior a 140 × $90 \mathrm{mmHg}$. Destes dez, dois apresentavam todos os critérios expostos para hipertensão refratária, ou seja, três classes em dose máxima, sendo uma delas diurético; dos oito restantes, quatro não utilizavam diurético, dois utilizavam apenas duas classes de drogas e dois, apesar de utilizarem três classes de anti-hipertensivos, não utilizavam as doses máximas preconizadas.

\section{DıscussÃo}

O trabalho em questão é um estudo caso-controle (pareado por idade e sexo) que verifica as características clínicas associadas ao desenvolvimento da nefroangioesclerose hipertensiva "benigna" confirmada por biópsia renal. Identificou-se o tempo de hipertensão arterial e a maior pressão de pulso como diferenciais entre os

\begin{tabular}{lccc} 
Tabela 2 & $\begin{array}{l}\text { VARIÁVEIS DISCRETAS COMPARANDO PACIENTES COM NEFROANGIOESCLEROSE HIPERTENSIVA E GRUPO } \\
\text { ConTROLE DE HIPERTENSOS PAREADO POR IDADE E SEXO }\end{array}$ & \\
& $\begin{array}{c}\text { Grupo Nefroangioesclerose } \\
(\mathrm{n}=15)\end{array}$ & $\begin{array}{c}\text { Grupo Controle } \\
(\mathrm{n}=15)\end{array}$ & $\mathrm{p}$ \\
\hline Sexo (F:M) & $2: 13$ & $2: 13$ & 0,87 \\
Raça (B:N) & $13: 2$ & $14: 1$ & 0,56 \\
Tabagismo (sim:não:prévio) & $2: 6: 6$ & $3: 5: 4$ & 0,86 \\
Diabetes (sim:não) & $3: 10$ & $2: 11$ & 0,63 \\
Diurético (sim:não) & $13: 2$ & $8: 7$ & 0,05 \\
Tiazídico (sim:não) & $2: 13$ & $7: 8$ & 0,11 \\
Furosemida (sim:não) & $12: 3$ & $1: 14$ & $<0,01$ \\
Bloqueador de cálcio (sim:não) & $5: 10$ & $1: 14$ & 0,07 \\
Beta-bloqueador (sim:não) & $3: 12$ & $2: 13$ & 0,64 \\
Simpatolítico (sim:não) & $7: 8$ & $2: 13$ & 0,05 \\
Vasodilatador (sim:não) & $1: 14$ & $0: 15$ & 0,32 \\
BRA (sim:não) & $2: 13$ & $1: 14$ & 0,56 \\
IECA (sim:não) & $4: 11$ & $5: 10$ & 0,70 \\
Duplo bloqueio (sim:não) & $1: 14$ & $1: 14$ & 1,00
\end{tabular}

F: feminino; M: masculino; B: brancos; N: não brancos; BRA: bloqueadores do receptor de angiotensina; IECA: inibidores da enzima conversora do angiotensinogênio. 
que desenvolveram a lesão renal hipertensiva e o Grupo Controle, mostrando a importância de fatores hemodinâmicos na gênese dessa alteração. Identificou-se também a elevação do ácido úrico e da glicemia, o que sugere a participação de fatores metabólicos na modulação do efeito lesivo da pressão arterial sobre o rim. Há que se lembrar que esses pacientes apresentavam nefroangioesclerose hipertensiva confirmada por biópsia renal; dessa maneira, tem-se certeza de que não havia pacientes com nefropatia diabética (apesar de alguns pacientes serem diabéticos) ou glomerulopatias.

Neste estudo, diferentemente da literatura, não se observou maior frequência de raça negra entre os portadores de nefroangioesclerose hipertensiva. Esse fato pode ter sua explicação na miscigenação frequente que ocorre no Brasil: é possível que os fenotipicamente brancos possuam componentes genéticos da raça negra, o que lhes confere uma predisposição maior à lesão renal hipertensiva, minimizando, assim, as potenciais diferenças.

$\mathrm{O}$ maior número de classes de anti-hipertensivos e maior frequência de uso de simpatolíticos e diuréticos entre os pacientes que apresentavam nefroangioesclerose hipertensiva deve ter sua origem na hipertensão arterial mais grave com necessidade, mais frequentemente, do uso dessas classes de medicações. Há que se recordar que os simpatolíticos centrais não constituem medicações de primeira linha na HA e são utilizados, via de regra, nos casos de HA refratária. Esse fato ressalta a gravidade da hipertensão nesses pacientes, uma vez que, apesar do uso de potentes anti-hipertensivos, estes se mantiveram com pressão de pulso superior.

As alterações laboratoriais do presente estudo entre os portadores de nefroangioesclerose hipertensiva lembram as observadas nos portadores de síndrome metabólica: maior elevação de glicemia e de ácido úrico (deve-se lembrar que, apesar de frequentemente associada à síndrome metabólica, a elevação da uricemia não constitui critério diagnóstico dessa síndrome). Um estudo experimental demonstrou o papel da síndrome metabólica na lesão podocitária de ratos espontaneamente hipertensos. Esse estudo verificou que o aumento da aldosterona, causado por adipocinas ainda não identificadas, e o estresse oxidativo observados nesses animais podem mediar as alterações renais. $\mathrm{O}$ estresse oxidativo e as lesões foram revertidos pelo uso do eplerenone. ${ }^{7}$

Uma coorte transversal brasileira demonstrou a associação entre aumento de ácido úrico e a presença de nefroangioesclerose hipertensiva definida clinicamente. ${ }^{8}$ Um estudo experimental indica que a hiperuricemia leve induz hipertensão sistêmica e injúria renal sem deposição de cristais, o que deve ser relacionado à disfunção endotelial e ativação do sistema renina. ${ }^{9}$
Os achados do presente estudo, no qual os portadores de glomeruloesclerose hipertensiva apresentavam valor elevado de pressão de pulso e de ácido úrico, podem ser justificados pelo fato de que o risco renal é estreitamente relacionado com a rigidez arterial ${ }^{10}$ assim, a pressão de pulso e não a pressão sistólica ou diastólica diferiu entre os grupos. As lesões vasculares arterioescleróticas no rim, relacionadas com a hipertensão, afetam primariamente as arteríolas pré-glomerulares, resultando em alterações isquêmicas nos glomérulos e nas estruturas pós-glomerulares. $\mathrm{O}$ aumento da rigidez das arteríolas pré-glomerulares propicia a transmissão da onda de pulso para o interior do glomérulo com consequente lesão. ${ }^{11-14}$ Esse aumento pode ser demonstrado em modelos experimentais por indução de hiperuricemia, tendo em vista que o ácido úrico produz o enrijecimento das arteríolas renais pré-glomerulares. ${ }^{15}$

No diabetes tipo II, a resistência insulínica associase a lesão renal. Mesmo em fases precoces da síndrome metabólica, antes até do aparecimento do diabetes, essa resistência pode induzir lesão. Com o tempo, os glomérulos perdem sua integridade funcional, resultando em defeitos de filtração, aumento da permeabilidade glomerular e aparecimento de microalbuminúria. ${ }^{16}$ Esse fato justifica a presença de hiperglicemia subclínica nos pacientes com nefroangioesclerose hipertensiva do presente estudo. É interessante notar que a frequência de diabéticos foi semelhante nos dois grupos. Ainda, analisando-se apenas os não diabéticos, os resultados do presente estudo não se alteram significativamente (dados não apresentados).

Devem-se reconhecer algumas limitações deste estudo. A avaliação da proteinúria foi realizada apenas por exame de urina I no Grupo Controle; assim, alguns pacientes deste grupo poderiam não ter sido corretamente diagnosticados. Entretanto, esse fato tenderia a atenuar as diferenças entre os grupos, o que apenas reforça as conclusões apresentadas. $\mathrm{O}$ pequeno número da amostra foi suficiente para demonstrar as diferenças quanto à pressão de pulso entre os dois grupos. Entretanto, não foram detectadas diferenças estatisticamente significantes quanto às variáveis índice de massa corpórea e triglicérides; assim, com um número maior de casos, não há como excluir a possibilidade de detectarmos diferença estatística dessas variáveis entre os grupos. Dessa maneira, apesar de o número pequeno da amostra deste estudo ter dificultado a confirmação da presença de diferenças significantes de algumas variáveis, os dados positivos obtidos (diferenças quanto à pressão de pulso, tempo de HA, ácido úrico, creatinina, glicemia, frequência de uso de diuréticos e de simpatolíticos) não perdem sua validade e importância. Ainda, apesar do número reduzido de casos avaliados no 
presente estudo, a quase totalidade dos estudos prévios avaliou casuísticas com diagnóstico apenas presuntivo de nefroangioesclerose hipertensiva. O número pequeno de casos acumulados em tantos anos justifica-se, pois, rotineiramente, não são realizadas biópsias em pacientes com diagnóstico presuntivo de nefroangioesclerose hipertensiva. Tal diagnóstico é feito pela exclusão de outras patologias primárias; dessa maneira, esse exame só é feito quando há dúvida diagnóstica.

As diferenças observadas com relação à proteinúria e função renal podem ter sido ocasionadas pela falácia de Berkson, ou viés de admissão, na qual pacientes com determinado fator de risco são selecionados para o estudo, tendo em vista que os critérios diagnósticos para indicação da biópsia renal incluíam proteinúria significativa (acima de 2,0 g) e piora progressiva da função renal. Entretanto, é pouco provável que pacientes com nefroangioesclerose hipertensiva não apresentem proteinúria ou piora progressiva da função renal. Estudos caso-controle estão sujeitos ao viés de sobrevivência seletiva (prevalência/incidência) ou viés de Neyman: casos prevalentes representam sobreviventes da doença em questão e como sobreviventes podem ser atípicos com relação à exposição do fator a ser testado: apresentariam fatores de risco abrandados com terapêutica mais adequada. Assim, o viés de sobrevivência seletiva tenderia a subestimar a pressão arterial, acido úrico e glicemia no grupo com nefroesclerose que apresentou maiores valores dessas variáveis, o que, na verdade, vem reforçar a conclusão deste estudo. Esse tipo de viés pode explicar a associação entre maior uso de anti-hipertensivos e nefroangioesclerose, ou seja, não é o uso dos anti-hipertensivos que causou a nefroesclerose hipertensiva, mas a hipertensão arterial mais grave nesse grupo fez com que os pacientes usassem mais anti-hipertensivos.

Este trabalho corrobora observações prévias e tem como diferencial em relação à literatura apresentar uma coorte com confirmação anatomopatológica da nefroangioesclerose hipertensiva. Assim, os dados obtidos permitem corroborar a ideia de que uma maior intensidade da hipertensão exercida por maior tempo e modulada por fatores metabólicos distinguem as pessoas que evoluíram para nefroangioesclerose hipertensiva, o que incentiva o controle estrito do nível de pressão arterial e de variáveis metabólicas para a prevenção da deterioração renal na hipertensão arterial.

\section{RefERÊNCIAS}

1. VI Diretrizes Brasileiras de Hipertensão. J Bras Nefrol 2010; 32(Supl):S1-S64.

2. Malta DC, Moura L, Souza FM, Rocha FM, Fernandes FM. Doenças crônicas não-transmissíveis: mortalidade e fatores de risco no Brasil, 1990 a 2006. Brasília: Ministério da Saúde; 2009. p. 337-62.

3. Romão Jr JE. Doença renal crônica: definição, epidemiologia e classificação. J Bras Nefrol 2004;26(Supl):1-3.

4. Shulman NB, Ford CE, Hall WD, Blaufox MD, Simon D, Langford HG et al. Prognostic value of serum creatinine and effect of treatment of hypertension on renal function. Results from the hypertension detection and follow-up program. The Hypertension Detection and Follow-up Program Cooperative Group. Hypertension 1989;13(Suppl):I80-93.

5. Caetano ER, Zats R, Saldanha L, Praxedes J. Hypertensive Nephrosclerosis as a Relevant Cause of Chronic Renal Failure. Hypertension 2001;38:171-6.

6. Marín R, Gorostidi M, Fernández-Vega F, AlvarezNavascuéz R. Systemic and glomerular hypertension and progression of chronic renal disease: the dilemma of nephrosclerosis. Kidney Int 2005:(Suppl):S52-6

7. Nagase M, Yoshida S, Shibata S, Nagase T, Gotoda T, Ando $\mathrm{K}$ et al. Enhanced aldosterone signaling in the early nephropathy of rats with metabolic syndrome: possible contribution of fat-derived factors. J Am Soc Nephrol 2006;17:3438-46.

8. Borges R, Hirota A, Quinto B, Ribeiro A, Zanella M, Batista M. Uric Acid as a Marker for Renal Dysfunction in Hypertensive Women on Diuretic and Nondiuretic Therapy. J Clin Hypertens (Greenwich) 2009; 11:253-9.

9. Mazzali M, Hughes J, Kim YG, Jefferson JA, Kang DH, Gordon KL et al. Elevated uric acid increases blood pressure in the rat by a novel crystal-independent mechanism. Hypertension. 2001;38:1101-6.

10. Safar ME, London GM, Plante GE. Arterial stiffness and kidney function. Hypertension. 2004;43:163-8.

11. Tracy RE, Ishii T. What is 'nephrosclerosis'? Lessons from the US, Japan, and Mexico. Nephrol Dial Transplant 2000;15:1357-66.

12. Tracy RE, Malcom GT, Oalmann MC, Qureshi U, Ishii T, Velez-Duran M. Renal microvascular features of hypertension in Japan, Guatemala, and the United States. Arch Pathol Lab Med 1992;116:50-5.

13. Tracy RE, Newman WP 3rd, Wattigney WA, Srinivasan SR, Strong JP, Berenson GS. Histologic features of atherosclerosis and hypertension from autopsies of young individuals in a defined geographic population: the Bogalusa Heart Study. Atherosclerosis 1995;116:163-79.

14. Tracy RE, Parra D, Eisaguirre W, Torres Balanza RA. Influence of arteriolar hyalinization on arterial intimal fibroplasia in the renal cortex of subjects in the United States, Peru, and Bolivia, applicable also to other populations. Am J Hypertens 2002;15:106473.

15. Johnson R, Sega M, Srinivas T, Ejaz A, Mu W, Roncal $\mathrm{C}$ et al. Essential hypertension, progressive renal disease, and uric acid: a pathogenetic link? J Am Soc Nephrol 2005;16:1909-19.

16. Alexander MP, Patel TV, Farag YM, Florez A, Rennke HG, Singh AK. Kidney pathological changes in metabolic syndrome: a cross-sectional study. Am J Kidney Dis 2009;53:751-9. 